\title{
Beat-to-beat Complexity Analysis Before the Onset of Ventricular Tachycardia
}

\author{
N Wessel ${ }^{1,2}$, N Marwan $^{1}$, A Schirdewan $^{2}$, J Kurths $^{1}$ \\ ${ }^{1}$ Institute of Physics, University of Potsdam, Germany \\ ${ }^{2}$ Franz-Volhard-Hospital, Helios-Clinics, Charité, Humboldt University Berlin, Germany
}

\begin{abstract}
We present recently introduced new recurrence plot based measures of complexity and illustrate their potential with applications to the logistic map and heart rate variability data. These new measures make the identification of chaos-chaos transitions possible and identify laminar states. The application to the heart rate variability data detects and quantifies the laminar phases before a life-threatening cardiac arrhythmia occurs; thereby facilitating a possible prediction of such an event. A comparison to the previous applied methods from symbolic dynamics and the finite-time growths rates is given.
\end{abstract}

\section{Introduction}

Numerous scientific disciplines use data analysis techniques to get an insight into the complex processes observed in nature which show generally a nonstationary and complex behavior. As these complex systems are characterized by different transitions between regular, laminar and chaotic behaviors, the knowledge of these transitions is necessary for understanding the process. Linear approaches of time series analysis are often not sufficient and most of the nonlinear techniques, such as fractal dimensions or Lyapunov exponents, suffer from the curse of dimensionality and require rather long data series.

To overcome the difficulties with nonstationary and rather short data series, the method of recurrence plots $(R P)$ has been introduced [1]. An additional quantitative analysis of recurrence plots has been developed to detect transitions (e.g. bifurcation points) in complex systems [2]. However, these measures can identify only transitions between chaos and order. Therefore, we present here three other measures basing on RPs and demonstrate their potentials for a prototypical nonlinear model and for cardiac data [3].

\section{Recurrence plots}

The method of recurrence plots (RP) was firstly introduced to visualize the time dependent behavior of the dynamics of systems, which can be pictured as a trajectory $\vec{x}(t)=\vec{x}_{i} \in R^{n}(i=1, \ldots, N, t=i \Delta t$, where $\Delta t$ is the sampling rate) in the $n$-dimensional phase space [1]. It represents the recurrence of the phase space trajectory to a certain state, which is a fundamental property of deterministic dynamical systems. The main step of this visualization is the calculation of the $N \times N$-matrix

$$
\mathbf{R}_{\mathbf{i}, \mathbf{j}}:=\boldsymbol{\Theta}\left(\varepsilon-\left\|\tilde{\mathbf{x}}_{\mathbf{i}}-\tilde{\mathbf{x}}_{\mathbf{j}}\right\|\right), \quad \mathbf{i}, \mathbf{j}=\mathbf{1} \ldots \mathbf{N}
$$

where $\varepsilon$ is a cut-off distance, $\|\cdot\|$ a norm (e.g. $L_{2}$ or $L_{\infty}$ norm; in this work the $L_{2}$ norm is used) and $\Theta(x)$ the Heaviside function. The phase space vectors for one-dimensional time series $u_{i}$ from observations can be reconstructed by using the Taken's time delay method $\vec{x}_{i}=\left(u_{i}, u_{i+\tau}, \ldots, u_{i+(m-1) \tau}\right)$ with dimension $m$ and delay $\tau$, whereby the dimension $m$ can be estimated by using methods basing on false nearest neighbors. The binary values in $R_{i, j}$ can be simply visualized by a matrix plot with the colors black (1) and white (0). The recurrence plot exhibits characteristic large-scale and smallscale patterns which are caused by typical dynamical behavior [1, 4], e. g. diagonals (similar local evolution of different parts of the trajectory) or horizontal and vertical black lines (state does not change for some time). Recently introduced extensions to cross recurrence plots use the diagonal structures and their distortions, respectively, for finding similarities and time transfer functions between two different systems [5]. Zbilut and Webber have developed the recurrence quantification analysis (RQA) to quantify an RP [4]. They defined measures using the recurrence point density and diagonal structures in the recurrence plot, e.g. the recurrence rate $R R$ (percent recurrences, density of recurrence points), the determinism $D E T$ (ratio of recurrence points forming diagonal structures to all recurrence points), the maximal length of diagonal structures $L_{\max }$ (or their averaged length $L$ ). A theoretical approach to the RQA including the effect of observational noise was recently published by Thiel et al. [6]. Trulla et al. have applied the RQA in order to find transitions in dynamical systems [2]. They have showed, that the RQA measures are able to find transitions between chaos and order (periodical states). But they could not find chaoschaos transitions, which are very typical in dynamical systems. 

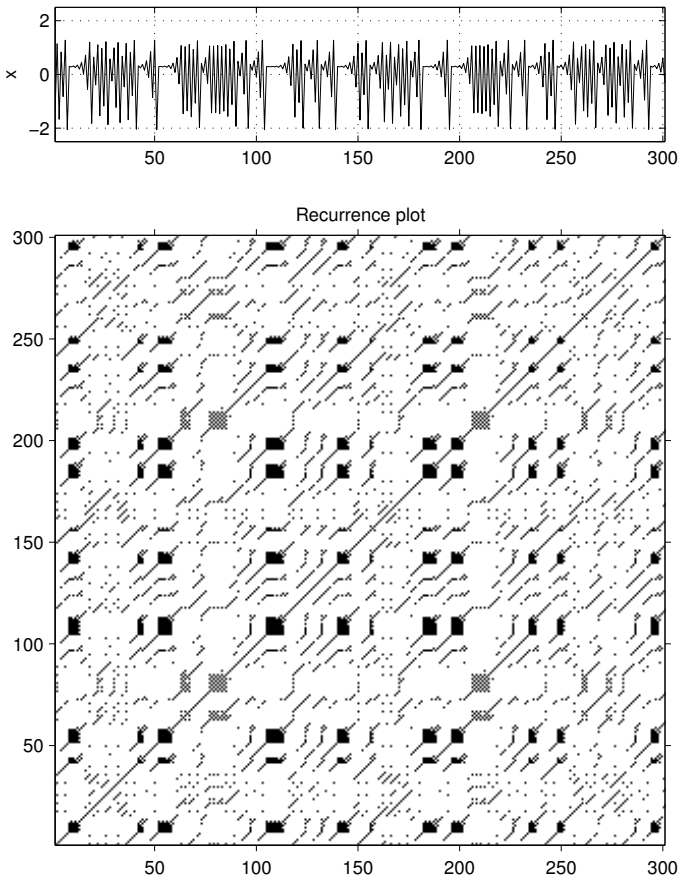

Figure 1. Exemplary recurrence plot of the logistic map for the band merging $a=3.679$; RP parameters are $m=1$, $\tau=1$ and $\varepsilon=0.1 \sigma$.

\section{Measures of complexity}

Therefore, we have recently introduced two additional measures which are based on the vertical structures in the RP [3]. We define these measures analogous to the definition of $D E T$ and $L$ (and $L_{\max }$ ), but we consider the distribution $P(v)$ of the length of the vertical structures in the RP. First, the laminarity $L A M$

$$
L A M:=\frac{\sum_{v=2}^{N} v P(v)}{\sum_{v=1}^{N} v P(v)},
$$

is the ratio of recurrence points forming vertical structures to all recurrence points and represents the probability of occurrence of laminar states in the system, but it does not describe the length of these laminar phases. It will decrease if the RP consists of more single recurrence points than vertical structures. Next, the trapping time $T T$

$$
T T:=\frac{\sum_{v=2}^{N} v P(v)}{\sum_{v=2}^{N} P(v)},
$$

is the averaged length of the vertical structures. The measure $T T$ contains information about the amount and the length of the laminar phases. Finally, we use the maximal length of the vertical structures in the RP

$$
V_{\max }:=\max \left(\left\{v_{l} ; l=1,2, \ldots L\right\}\right)
$$
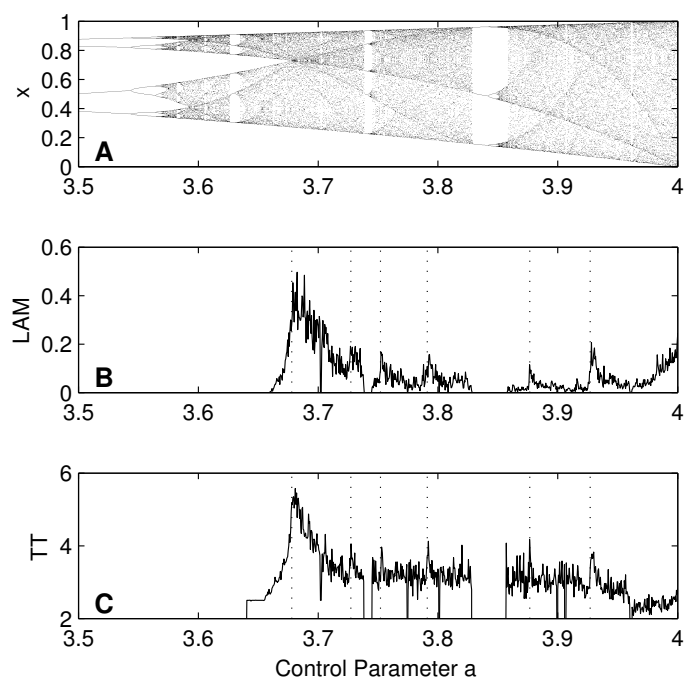

Figure 2. Laminarity (B) and trapping time (C) of time series gained from the logistic map for various control parameters (A). These measures reveal laminar and intermittent states. The vertical dotted lines show a choosing of points of band merging and laminar behavior ( $a=3.678,3.727,3.752,3.791,3.877,3.927)$.

as a measure, which is the analogue to the standard RQA measure $L_{\max }$. The distinction between these measures and the traditional RQA measures is their ability to find transitions between chaos and chaos [3].

\section{Application to the logistic map}

In order to illustrate the potentials of $L A M, T T$ and $V_{\max }$, we firstly apply them to the logistic map $x_{n+1}=a x_{n}\left(1-x_{n}\right)$, especially the interesting range of the control parameter $a \in[3.5,4]$. We generate for each control parameter $a$ a separate time series. In the analyzed range of $a$ various regimes and transitions between them occur, e. g. accumulation points, periodic and chaotic states, band merging points, period doublings, inner and outer crisis [7]. We compute the RP with a cut-off distance of $\varepsilon=0.1$ (in units of the standard deviation $\sigma$ ); an embedding is not necessary here (i.e. $m=1$ and $\tau=$ 1 ). The cut-off distance $\varepsilon$ is selected to be 10 percent of the diameter of the reconstructed phase space. Smaller values would lead to a better distinction of small variations (e. g. the range before the accumulation point consists of small variations), but the recurrence point density decreases in the same way and thus the statistics of continuous structures in the RP becomes soon insufficient. For various values of the control parameter $a$ the RPs exhibits specific features (an exemplary RP is shown in Fig. 1). Periodic states cause continuous and periodic diagonal lines in the 

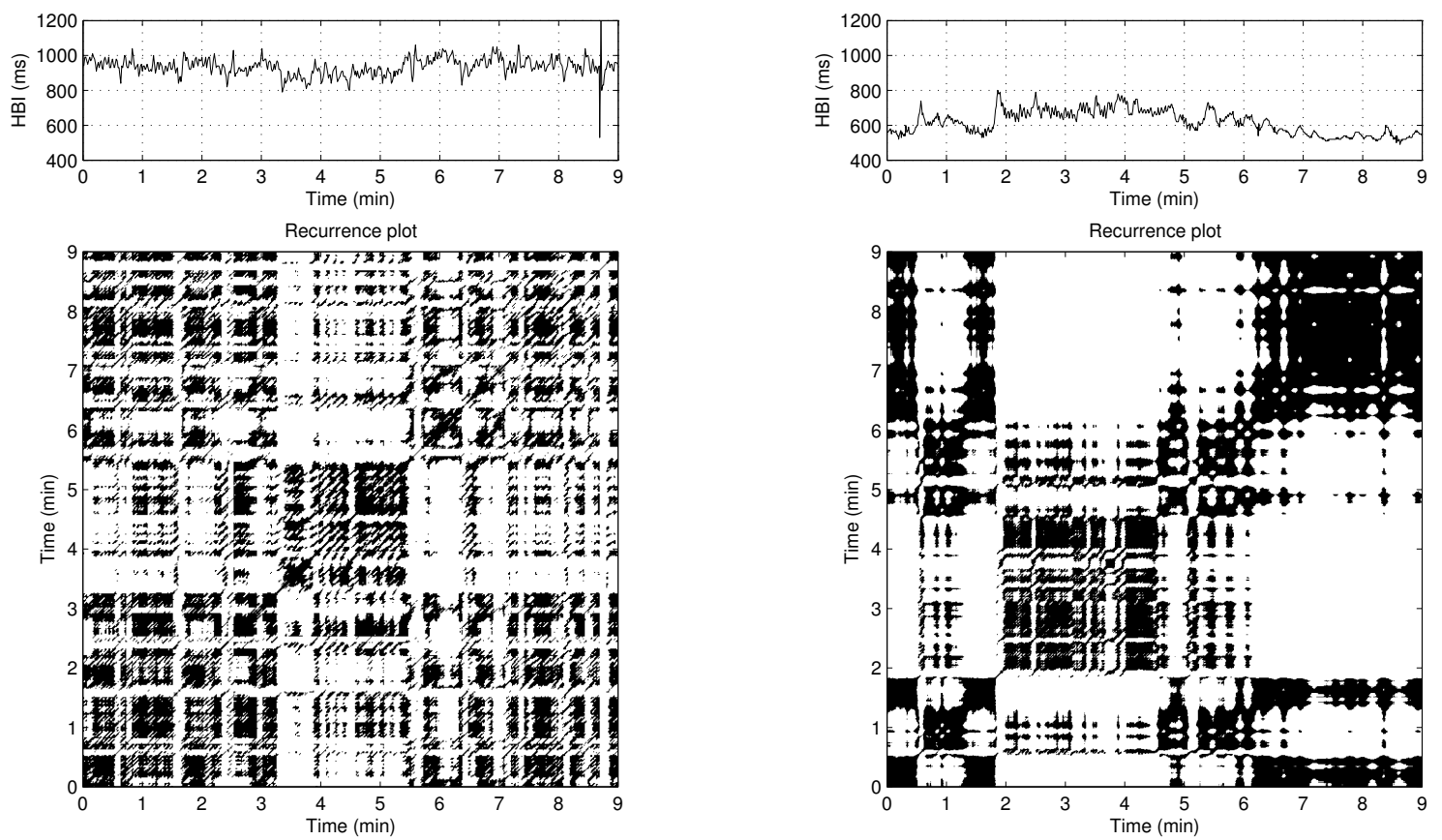

Figure 3. Recurrence plots of the heart beat interval (HBI) time series at a control time (left) and before a VT (right) with $m=6$ and $\varepsilon=170$. The RP before a life-threatening arrhythmia is characterized by big black rectangles whereas the RP from the control series shows only small rectangles.

RP but no vertical or horizontal lines. Band merging points and inner crisis represent states with short laminar behavior and cause vertically and horizontally spread black areas in the RP. Fully developed chaotic states $(a=4)$ cause a rather homogeneous RP with numerous single points and rare short diagonal or vertical lines. Therefore, the measures $L A M, T T$ and $V_{\max }$, which base on these vertical structures, find the periodic-chaotic/ chaoticperiodic transitions as well as the laminar states (Fig. 2 B, C). Since vertical lines occur much more frequently at inner crisis and band merging points (i.e. laminar states) than in other chaotic regimes, $L A M$ and $T T$ grows up at those points. Although $V_{\max }$ also reveals laminar states, it is quite different from the other two measures, because it gives the maximum of all of the durations of the laminar states [3]. Hence, the vertical length based measures yield periodic-chaotic/ chaotic-periodic as well as chaos-chaos transitions (laminar states).

\section{Application to HRV data}

A major challenge in biological physics is the analysis of cardiac time series. Heart rate variability (HRV) typically shows a complex behavior and it is difficult to identify disease specific patterns. Implantable cardioverter defibrillators (ICD) are a safe and effective treatment for ventricular tachycardia or fibrillation (VT). These fatal cardiac arrhythmias are the main factors triggering sudden cardiac death. A fundamental challenge in cardiology is to find early signs of VT in patients with an ICD based on HRV data (e.g. [8]). Recently studies applied standard methods, methods basing on symbolic dynamics as well as finite-time growth rates to the HRV parameters from time and frequency domain $[8,9,10,11,12]$. The defibrillators used in this study are able to store at least 1000 beat-tobeat intervals prior to the onset of VT $(10 \mathrm{~ms}$ resolution, Biotronik), corresponding to approximately 9-15 minutes. We studied the ICD stored beat-to-beat intervals before the onset of $24 \mathrm{VT}$ episodes and at 24 control intervals without VT in 17 ICD patients of the Franz-VolhardHospital with severe congestive heart failure. The beatto-beat intervals of the VT at the end of the time series were removed from the tachograms so that we analyzed only the dynamics occurring immediately prior to VT. We calculate all standard RQA parameters described in [4] as well as the new measures $L A M, T T$ and $V_{\max }$ for different embedding dimensions $m$ and vicinity threshold radii $\varepsilon$. We find differences between both groups of data for several of the parameters mentioned above. However, the most significant parameters are $V_{\max }$ and $L_{\max }$ for rather large radii (Tab. 1). The vertical line length $V_{\max }$ is more powerful in significantly discriminating both groups than the diagonal line length $L_{\max }$, as can be recognized by the higher $p$-values for $V_{\max }$ (Tab. 1). The RP before a 
life-threatening arrhythmia is characterized by large black rectangles, whereas the RP from the control series shows only small rectangles (Fig. 3).

Table 1. Results of $L_{\max }$ and $V_{\max }$ shortly before VT and at control time, nonparametric Mann-Whitney U-test, $p$ - significance; $*-p<0.05$; $* *-p<0.01$; ns - not significant $p \geq 0.05$ )

\begin{tabular}{rrrrcccc}
\hline \multirow{2}{*}{$m$} & $\varepsilon$ & \multicolumn{3}{c}{$\left\langle L_{\max }\right\rangle$} & \multicolumn{3}{c}{$\left\langle V_{\max }\right\rangle$} \\
& & $\mathrm{VT}$ & $\mathrm{Ctr}$. & $p$ & $\mathrm{VT}$ & $\mathrm{Ctr}$. & $p$ \\
\hline 3 & 77 & 396.6 & 261.5 & $\mathrm{~ns}$ & 261.4 & 169.2 & $*$ \\
6 & 110 & 447.6 & 285.5 & $*$ & 283.7 & 179.5 & $* *$ \\
9 & 150 & 504.6 & 311.6 & $*$ & 342.4 & 216.1 & $* *$ \\
12 & 170 & 520.7 & 324.7 & $*$ & 353.5 & 215.1 & $* *$ \\
\hline
\end{tabular}

In Tab. 2 a comparison to the previous applied methods from symbolic dynamics and the finite-time growths rates is given [11]. The highest correlation was found for the recurrence rate and the Shannon-Entropy of the histogram: the higher the entropy of the histogram the lower the RR. For the parameter DET a relative high correlation was found to 'Shannon' whereas $V_{\max }$ is only moderately correlated.

Table 2. Correlation coefficients of the RQA parameters ( $m=12, \varepsilon=170$ ) to the previous applied measures 'Shannon' (the Shannon-Entropy of the histogram), 'FWShannon' (the Shannon-Entropy of the word distribution), 'PLVAR10' (symbolic dynamics parameter) as well as the finite time growth rates $\lambda_{3}, \lambda_{6}$ and $\lambda_{9}$. All coefficients are significantly different from zero $p<0.05$ (except $R R$ vs. $\lambda_{9}$ ).

\begin{tabular}{cccc}
\hline & $R R$ & $D E T$ & $\left\langle V_{\max }\right\rangle$ \\
\hline Shannon & -0.85 & -0.77 & -0.49 \\
FWshannon & -0.41 & -0.49 & -0.41 \\
PLVAR10 & 0.49 & 0.73 & 0.48 \\
$\lambda_{3}$ & -0.71 & -0.74 & -0.57 \\
$\lambda_{6}$ & -0.36 & -0.63 & -0.49 \\
$\lambda_{9}$ & 0.00 & -0.26 & -0.27 \\
\hline
\end{tabular}

\section{Conclusions}

We have demonstrated that our new three measures of complexity basing on recurrence plots are able to identify chaos-chaos transitions and epochs of laminar behavior. The application of these measures to heart rate variability data, has shown, that they are able to detect and quantify laminar phases before a life-threatening cardiac arrhythmia and, thus, may predict such an event [3]. These findings can be of importance for the therapy of malignant cardiac arrhythmias. Limitations of this analysis are the relatively small number of time series and the reduced statistical analysis (no subdivisions concerning age, sex, heart disease,
VT time). For this reason, these results have to be validated on prospective data from the MARITA multicentric study were 9000 RR-intervals before a VT are stored. A download of a Matlab implementation is available at: tocsy.agnld.uni-potsdam.de.

\section{References}

[1] Eckmann JP, Kamphorst SO, Ruelle D. Recurrence Plots of Dynamical Systems. Europhysics Letters 1987;5:973-977.

[2] Trulla LL, Giuliani A, Zbilut JP, et al. Recurrence quantification analysis of the logistic equation with transients. Physics Letters A 1996;223(4):255-260.

[3] Marwan N, Wessel N, Meyerfeldt U, et al. Recurrence Plot Based Measures of Complexity and its Application to Heart Rate Variability Data. Physical Review E 2002; 66(2):026702.

[4] Webber Jr. CL, Zbilut JP. Dynamical assessment of physiological systems and states using recurrence plot strategies. Journal of Applied Physiology 1994;76(2):965973.

[5] Marwan N, Kurths J. Nonlinear analysis of bivariate data with cross recurrence plots. Physics Letters A 2002;302(56):299-307.

[6] Thiel M, Romano MC, Kurths J, et al. Influence of observational noise on the recurrence quantification analysis. Physica D 2002;171(3):138-152.

[7] Collet P, Eckmann JP. Iterated maps on the interval as dynamical systems. Basel Boston Stuttgart: Birkhäuser, 1980.

[8] Pruvot E, Thonet G, Vesin J, et al. Heart rate dynamics at the onset of ventricular tachyarrhythmias as retrieved from implantable cardioverter-defibrillators in patients with coronary artery disease. Circulation 2000;101:2398-2404.

[9] Kurths J, Voss A, Witt A, et al. Quantitative analysis of heart rate variability. Chaos 1995;5:88-94.

[10] Voss A, Kurths J, Kleiner HJ, et al. The application of methods of non-linear dynamics for the improved and predictive recognition of patients threatened by sudden cardiac death. Cardiovasc Res 1996;31:419-433.

[11] Wessel N, Ziehmann C, Kurths J, et al. Short-term forecasting of life-threatening cardiac arrhythmias based on symbolic dynamics and finite-time growth rates. Physical Review E 2000;61:733-739.

[12] Meyerfeldt U, Wessel N, Schutt $\mathrm{H}$, et al. Heart rate variability before the onset of ventricular tachycardia: differences between slow and fast arrhythmias. Int J Cardiol 2002;84:141-151.

Address for correspondence:

Dr. Niels Wessel

University of Potsdam, Institute of Physics, PF 601553, D-14415 Potsdam, Germany niels@agnld.uni-potsdam.de 